\title{
A MEASUREMENT OF THE GRAVITATIONAL CONSTANT USING A CRYOGENIC TORSION PENDULUM
}

\author{
M.K. Bantel, E.C. Berg, and R.D. Newman \\ Department of Physics and Astronomy, University of California at Irvine \\ Irvine CA 92697-4575, USA \\ mbantel@uci.edu, eberg@uci.edu,rdnewman@uci.edu
}

\begin{abstract}
$\underline{\text { Abstract }}$
Final analysis is in progress of a measurement of $G$ made using a cryogenic torsion pendulum in the 'dynamic' (time-of-swing) mode. We have collected and analyzed data which will yield a $\mathrm{G}$ measurement with about 20 ppm statistical uncertainty. A value for $\mathrm{G}$ will be reported at CPEM 2002.
\end{abstract}

\section{Method}

We determine $\mathrm{G}$ by measuring the change in oscillation frequency of a thin-plate torsion pendulum due to a pair of ring-shaped source masses positioned alternately as indicated in figure 1 . The pendulum is in an evacuated chamber within a dewar filled with liquid helium; the source mass rings are suspended outside the dewar at room temperature. The pendulum's suspension point is maintained at $\sim 2.7 \mathrm{~K}$, controlled within $0.3 \mathrm{mK}$. The high $\mathrm{Q}$ afforded by the low temperature operation minimizes bias due to fiber anelasticity [1].
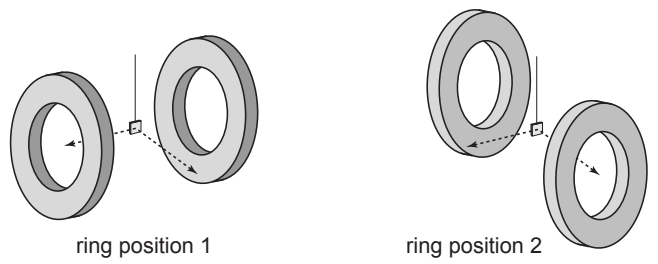

Figure 1: The pendulum and source mass rings. The pendulum is an 11 gram fused silica square plate 40x40x3 mm, suspend by a copper-beryllium fiber 20 $\mu \mathrm{m}$ diameter and $24 \mathrm{~cm}$ long. Each copper ring is 60 $\mathrm{kg}$ with dimensions: $52 \mathrm{~cm}$ OD, $32 \mathrm{~cm} \mathrm{ID,} 5 \mathrm{~cm}$ width.

The frequency shift due to the source mass rings may be expressed as: $\omega_{1}^{2}-\omega_{2}^{2} \cong K G J_{1}(2 A) / A$, where $\omega_{1}$ and $\omega_{2}$ are the pendulum's frequencies for the ring positions as indicated in figure $1, K$ is a geometric factor determined by the mass and dimensions of the pendulum and rings, $G$ is the gravitational constant, $J_{1}$ is a Bessel function, and $A$ is the oscillation amplitude of the pendulum. Thus by measuring the frequencies and amplitude of the pendulum, $K G$ may be determined. By restricting our knowledge of $K$ as we analyze our data, we are able to conduct a "blind" experiment minimizing the danger of bias.

\section{$\underline{\text { Apparatus }}$}

Figure 2 is a scale drawing showing the size and relative positions of the source mass rings, the pendulum and the evacuated pendulum housing within the liquid helium dewar. Details of this apparatus are reported elsewhere[2,3].

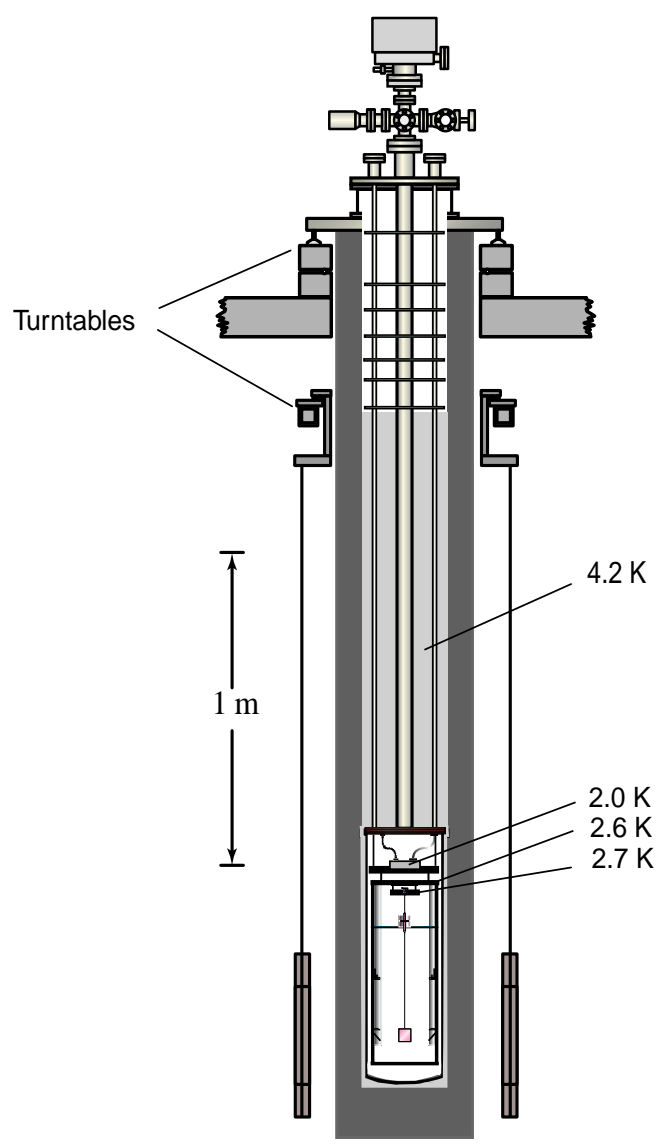

Figure 2: The cryogenic insert within the dewar, and suspended mass rings 


\section{Data}

We operate the pendulum at an oscillation amplitude near an extremum of $J_{1}(2 A) / A: 2.57,4.21,7.40$ radians. Data runs were made by setting the pendulum's amplitude to about $50 \mathrm{mrad}$ above one of the extrema, then allowing the pendulum to 'ring down' while alternately moving the source mass rings every 20 pendulum cycles (figure 3 ). We made 26 data runs with durations varying from 10 to 50 hours, totaling about 550 hours. For each of these runs, a value and uncertainty of $K G$ was determined (figure 4). Between sets of several runs, the source mass rings were rotated around their vertical and/or horizontal axes to average out possible asymmetries; four unique ring configurations were used. Occasional 'glitches' appearing in the pendulum's measured period (see figure 3 ) were removed in the determination of $K G$ values.

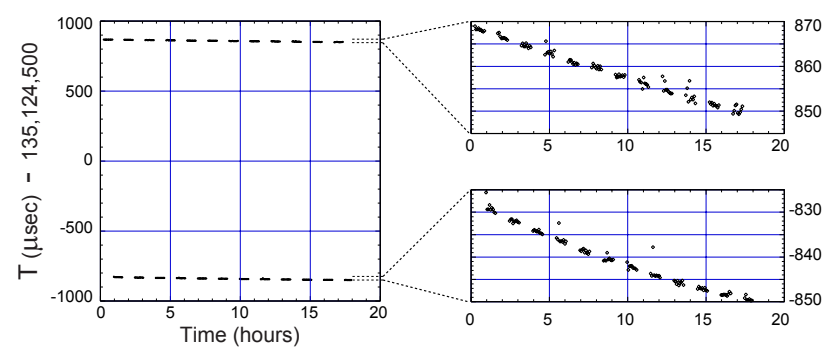

Figure 3: Typical data run (day 39) showing the period shift due to the source mass rings. The rings are moved every 20 pendulum cycles, at about 45 minutes intervals. Every two pendulum cycles a period $\mathbf{T}$ is determined and plotted; expanded plots are at right.

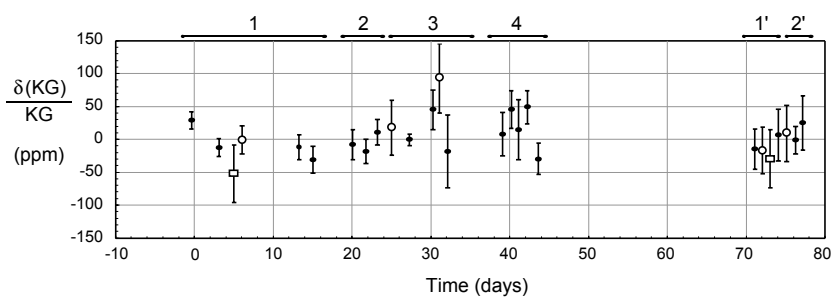

Figure 4: Determined $K G$ values for data runs from October to December 2000, expressed as their ppm deviation from their mean. For each run, the pendulum's oscillation amplitude was one of three nominal values: 2.57-solid circle, 4.21-open circle, and 7.40open square. Regions 1,2,3,4 correspond to different ring configurations. After day 45 the as-drawn fiber $(Q \sim 80,000)$ was replaced with a heat treated fiber of the same material $(Q \sim 120,000)$. Outliers, about $2 \%$ of the data, were excluded in the determination of $K G$ values.

\section{"Blind" Data Analysis}

To avoid possible bias in data selection and analysis as well as in evaluating uncertainties in measured quantities, we do all analysis in such a way that we do not know the value of $\mathrm{G}$ that is emerging at intermediate stages of the analysis. We do this by not allowing ourselves to know the exact result of the NIST measurement of the mass of our source mass rings until we are ready to announce a result. Thus our measurement analysis gives us measured values of the product KG and its uncertainty, but we do not know the exact value of $K$ and hence of $G$ until the final step of our analysis.

\section{Acknowledgments}

We are indebted to Los Alamos National Laboratory for the fabrication of the source mass rings, and to NIST (especially Z. Jabbour, H. Harary, and D. Everett)for much of the metrology. We are grateful to Roy Gephart and Pacific Northwest National Laboratory for providing a site and associated services. This work is funded by NSF grant PHY-9514944.

\section{References}

\section{$\underline{\text { References }}$}

[1] K. Kuroda, "Does the Time-of-Swing Method Give a Correct Value of the Newtonian Gravitational Constant?," Phys. Rev. Lett., Vol. 75, No. 15, pp. 2796-2798, October 1995.

[2] R.D. Newman and M.K. Bantel, "On Determining G Using a Cryogenic Torsion Pendulum," Meas. Sci. Technol., Vol. 10, pp. 445-453, 1999.

[3] M.K. Bantel and R.D. Newman, "A Cryogenic Torsion Pendulum: Progress Report," Class. Quantum Grav., Vol. 17, pp. 2313-2318, 2000. 